\title{
Determining standards of adequate veterinary care
}

$\mathrm{R}$ eddington State University had a relatively small animal-based research program but was registered with the USDA, maintained a written program of veterinary care, and held an NIH/ OLAW assurance. The school's attending veterinarian was Dr. Ron Meyers, a local private practitioner. He visited the vivarium once a month but came more often if an animal required medical attention.

One weekend, Meyers received a call from the vivarium manager who told him that a pig had gotten its foot wedged between the raised pen floor and the wall. When a caretaker opened the pen door to try to help the animal, the pig panicked, pulled out its leg, and sustained a deep cut above its hoof. Meyers came to the school and sedated the pig. He then cleaned, debrided, and sutured the wound, leaving instructions to call him if there were further problems or if the sutures did not fall out within two weeks. There were no further calls to Meyers so when he returned three weeks later for his regularly scheduled visit he was surprised to learn the pig had been euthanized. He was informed the wound became infected and the principal investigator said that because of the infection he could no longer use the animal as part of his ongoing experimentation. A few months afterwards, during a routine inspection, the USDA veterinary officer reviewed the IACUC meeting minutes in which the incident with the pig was described. As a result, the school was given a citation for inadequate veterinary care, stating that Meyers should have prescribed or at least discussed the use of antibiotics for the animal and he was negligent in not revisiting the animal to examine the wound, thereby leading to the animal's euthanasia. Meyers believed what he did was fully appropriate and asked the school to appeal the citation. He claimed that he could not be held to a standard of adequate veterinary care because there was no written standard of care, either in the federal regulations or the state's veterinary medical laws. Furthermore, he said that the euthanasia of the animal was a decision made by the investigator and neither he nor the school could be held responsible when an investigator chooses not to attempt further medical interventions.

Do you think the school should accept the citation and reevaluate its medical practices to try to prevent this type of problem from recurring? Appeal the citation? Or appeal the citation and concurrently reevaluate its practices?

\section{Jerald Silverman \\ University of Massachusetts Medical School, Worcester, Massachusetts, USA. \\ e-mail: Jerald.Silverman@umassmed.edu}

Published online: 24 September 2018 https://doi.org/10.1038/s41684-018-0153-1

\section{Better communication is advised}

$\mathrm{D}$ r. Meyers is correct in stating there are not explicit standards of adequate veterinary care provided by the USDA Animal Welfare Act and Animal Welfare Regulations ${ }^{1}$ (AWR) or state veterinary medical laws. Most states consider adequate standards of care to be what an average practitioner would do under similar circumstances, and the AWR definition of an attending veterinarian states, "a person who has graduated from a veterinary school...; has received training and/or experience in the care and management of the species being attended." Even though these statements are not specific, veterinarians are held to what are generally considered adequate standards of care; therefore, Dr. Meyers is incorrect in stating "he could not be held to a standard of adequate veterinary care." Standards of care are dictated by the experts in the field of veterinary medicine, up-to-date treatment protocols presented at continuing educations meetings, the standards taught at veterinary schools, and material found in current veterinary references.

The treatment provided by Dr. Meyers appears to adhere to the basic level of care for wound management. Additionally, he left explicit instructions that if the pig did not improve or became worse that he should be contacted. In our opinion, the school should appeal the citation as written and concurrently re-evaluate its practices. We do not feel Dr. Meyers was negligent in not revisiting the animal as he left explicit instructions that he should be contacted if problems occurred. He followed AWR, where Section 2.33 (b)(3) states, "a mechanism of direct and frequent communication is required so that timely and accurate information on problems of animal health, behavior, and well-being is conveyed to the attending veterinarian."'; unfortunately, the vivarium staff did not. If a citation was going to be given, it should have been issued for poor communication between the school and the veterinarian and not veterinary negligence as it was Dr. Meyer's professional opinion that the pig did not need antibiotics at the time. The Veterinary Medical Officer's citation of veterinary negligence is based purely on opinion, unless he/she has specific knowledge or expertise as to why antibiotics should have been used or discussed.

The school needs to re-evaluate a number of its practices/policies as well as its program of veterinary care. First, communication with the attending veterinarian needs to be improved. The school may want to require that before an animal is euthanized while under the care of the attending veterinarian the veterinarian must first be contacted, with the exception that in an emergency when the attending cannot be reached the animal may be euthanized. Second, the school should re-evaluate caretaker and PI training, since until the veterinarian determines a wound is infected it should not be assumed it is. The veterinarian should have been called to determine if the wound was infected and if so to have a discussion with the PI as to what options were available. Thirdly, the school's policy on euthanasia should be revisited to set up guidelines for situations such as this.

Laurie Serfilippi ${ }^{1 \star}$ and Danielle Pallman² ${ }^{1}$ Summit Ridge Farms, Susquehanna, Pennsylvania, USA. ${ }^{2}$ Calvert Laboratories, Inc., Scott Township, Pennsylvania, USA.

*e-mail:laurie@srfarms.com

Published online: 24 September 2018 https://doi.org/10.1038/s41684-018-0156-y

\footnotetext{
References

1. United States Department of Agriculture. Animal Welfare Act and Animal Welfare Regulations (2017) pgs. 32, 62.
} 